\title{
Chemical and genetic markers for identification of honey origin from its bee speciation
}

\begin{abstract}
The need for accurate and reliable methods for identification of honey origin is important for reducing honey fraud. This study has identified suitable chemical and genetic markers to determine the origin of honey from its bee source of Apis honey bees or Trigona stingless bee. In the chemical analysis, moisture, fructose, glucose, sucrose, free acidity, and colour intensity were chemical markers identified for differentiating honey by its bee origin. The use of principal component analysis (PCA) and hierarchical cluster analysis (HCA) on honey composition have successfully classified honey into groups of Apis and Trigona. In the DNAbased method, mitochondrial cytochrome c oxidase subunit I (COI) gene was used as a genetic marker to identify honey origin by its bee species accurately from the clear groupings and distinct clusters in phylogenetic trees. The genetic marker of COI gene is accurate and reliable for this identification as it has direct matching to its reference bee species. Incorporating both chemical and genetic markers affirm the identity of honey.
\end{abstract}

Keyword: Honey; Entomological origin; Chemical composition; Pattern recognition; DNA marker; Phylogenetic analysis 